\title{
STATISTICAL METHODS IN SONAR
}




\section{STUDIES IN SOVIET SCIENCE PHYSICAL SCIENCES}

\section{4}

THEORY OF PLASMA INSTABILITIES

Volume 1: Instabilities of a Homogeneous Plasma

A. B. Mikhailovskii

THEORY OF PLASMA INSTABILITIES

Volume 2: Instabilities of an Inhomogeneous Plasma

A. B. Mikhailovskii

NONEQUILIBRIUM STATISTICAL THERMODYNAMICS

D. N. Zubarev

REFRACTORY CARBIDES

G. V. Samsonov

WAVES AND SATELLITES IN THE NEAR-EARTH PLASMA

Ya. L. Al'pert

1975

ENVIRONMENTAL HAZARDS OF METALS:

Toxicity of Powdered Metals and Metal Compounds

I. T. Brakhnova

DOMAIN ELECTRICAL INSTABILITIES IN SEMICONDUCTORS

V. L. Bonch-Bruevich, I. P. Zvyagin, and A. G. Mironov

1976

THE ROTATING DISC ELECTRODE

Yu. V. Pleskov and V. Yu. Filinovskii

1977

THEORY OF TURBULENT PLASMA

V. N. Tsytovich

THEORETICAL FOUNDATIONS OF NONLINEAR ACOUSTICS

O. V. Rudenko and S. I. Soluyan

LASER-INDUCED DISCHARGE PHENOMENA

Yu. P. Raizer

LIOUID-PHASE OXIDATION OF OXYGEN-CONTAINING COMPOUNDS

E. T. Denisov, N. I. Mitskevich, and V. E. Agabekov

1978

ADHESION OF SOLIDS

B. V. Deryagin, N. A. Krotova, and V. P. Smilga

STATISTICAL METHODS IN SONAR

V. V. OI'shevskii

THE SILOXANE BOND

M. G. Voronkov, V. P. Mileshkevich, and Yu. A. Yuzhelevskii 


\section{STUDIES IN SOVIET SCIENCE}

\section{STATISTICAL \\ METHODS IN SONAR}

\section{V.V. Ol'shevskii}

Acoustics Institute

Academy of Sciences of the USSR Moscow, USSR

Technical Editor

David Middleton

University of Rhode /sland 
Ol'shevskii, Viktor Vladimirovich.

Statistical methods in sonar.

(Studies in Soviet science: Physical sciences)

Translation of Statisticheskie metody $v$ gidrolokatsii.

Bibliography: $p$.

Includes index.

1. Sonar. I. Title. II. Series

VM480.04713

$623.89^{\prime} 38$

78-18196

ISBN 978-1-4757-0476-1

ISBN 978-1-4757-0474-7 (eBook)

DOI 10.1007/978-1-4757-0474-7

The original Russian text, published by Sudostroenie in Leningrad in 1973, has been corrected by the author for the present edition. This translation is published under an agreement with the Copyright Agency of the USSR (VAAP).

\section{СТАТИСТИЧЕСКИЕ МЕТОДЫ В ГИДРОЛОКАЦИИ}

В. В. О.1ьшевски и

\section{STATISTICHESKIE METODY $\vee$ GIDROLOKATSII}

V. V. OI'shevskii

(C) 1978 Consultants Bureau, New York

Softcover reprint of the hardcover 1st edition 1978

A Division of Plenum Publishing Corporation

227 West 17th Street, New York, N.Y. 10011

All rights reserved

No part of this book may be reproduced, stored in a retrieval system, or transmitted, in any form or by any means, electronic, mechanical, photocopying, microfilming, recording, or otherwise, without written permission from the Publisher 


\section{FOREWORD}

Dr. V. V. 01'shevskii is perhaps most familiar to Western readers as the author of "Characteristics of Sea Reverberation," published in translation by Consultants Bureau (New York, 1967). The present book, "Statistical Methods in Sonar," is, in part, a sequel to the first book, where now the author's stated purpose is "to acquaint a broad range of specialists with the use of contemporary statistical methods for solving theoretical and applied sonar problems." As the author quite properly observes, the work is illustrative, devoted to a variety of relevant, specific technical problems from an analytical point of view, and is not in any way intended to be an all-inclusive treatise. Nevertheless, as the reader can verify subsequently, the author has succeeded in accomplishing his stated purpose. He has, moreover, provided us with a use$\mathrm{ful}$ and, in a number of instances, provocative work, which even five years after its original appearance retains its freshness and interest with material not to date covered in other books on the subject (for example, see Horton [\$0], Stephens [41]).*

In this Foreword we first concisely review the author's material, on a chapter-by-chapter basis, after which a short general critique is given. Attention is called to various topics of particular interest to the professional audience, as well as to a number of highlights which deserve the reader's notice (a few additional comments on the technical editing are then included).

First of all, the treatment is statistical and analytical. The principal specific general assumptions made throughout are:

\footnotetext{
*Numbers in brackets, here and in the text, refer to references in the Bibliography at the end of the book. Numbers beyond [36] have been added by the editor. (D.M.)
} 
(i) Gaussian (i.e., high-density Poisson processes) for scattering in the medium

(ii) independent, discrete scatterers ( $\$ 36)$

(iii) zero gradient oceans $(\nabla c=0)$

(iv) omnidirectional beam patterns ( $(136)$

(v) narrow-band signals (most1y)

(vi) essentially monostatic operation ( $\$ 44)$

The various pertinent statistics of received signal and reverberation are consequently: means, variances, and other second-order moments, such as correlation functions and spectra, along with the associated Gaussian and Rayleigh probability (density) distributions. Within these constraints a considerable body of results is then generated, along with a clear exposition of the approach, which, as expected, involves a direct application of now standard statistical communication-theoretic methods (see, for example, [12, 45-47]).

The nature of the material discussed may now be briefly described:

Chapter I: "Introductory Remarks": Here the author defines "sonar" for the purposes of this book as "active sonar," the "objects of research" as the "objects of detection," and the general aims of sonar usage here as involving detection, estimation, and classification of the objects of research. A sonar model is then specified in a general way. The main differences between sonar and radar are noted, with the following features unique to sonar: (i) much greater variability of the physical medium; (ii) more complex shapes (vis-à-vis the returned signal) of the objects of detection; (iii) presence of reverberation in the medium and from its boundaries; (iv) $c_{\text {acoustic }}<c_{E M}$ : the much slower speed of acoustic propagation in the medium, and the lower frequencies required because of absorption, lead to much smaller data rates and much greater restrictions on information flow in the channel. The author then states that the central tasks of analyzing and synthesizing sonar systems are to develop suitable "indices of effectiveness," e.g., appropriate probabilistic measures of performance. 
Chapter II: "Procedural Fundamentals of Experimental Research in Sonar": In this chapter the author presents a hierarchical ordering of qualitative and quantitative procedures for "zeroing-in" on the base model, i.e., the appropriate model of the particular sonar situation at hand, which is to be studied experimentally and theoretically.

Chapter III: "Probability Models of Hydroacoustic Signals": Two major approaches to signal propagation and scattering are distinguished here. These are: ( $i$ ), the so-called "wave models," which involve solving (a set of) appropriate wave equations (Langevin equations), with boundary and initial conditions and ensemble properties, the goal of which is to determine the probability distributions of the random variables in question; and (ii), "phenomenological models," which employ simplified signal structures and embed the detailed boundary conditions and ensemble properties in a set of appropriately introduced random parameters or processes, to yield scattered and received waveforms with characteristic amplitude and phase (or frequency) modulations, related phenomenologically to the various random scattering mechanisms and propagation conditions involved.

While method (i) is acknowledged to be conceptually more precise and physical, it has the chief disadvantage of of ten exclusive analytical complexity vis-à-vis the phenomenological approach, (ii), which is the approach adopted by the author in this book. Accordingly, the focus here is on "the statistical properties of measurable signals," and a further distinction is made between "canonical," "constructive," and "parametric" phenomenological models.

Chapter IV: "The Emitted Signals": This chapter is essentially an exercise in calculating ambiguity functions for various classes of emitted narrow-band signals (under the previously mentioned assumption of omnidirectional beams). While the results are not generally new, they provide a convenient set for reference (and later use in the book). Included here are ambiguity functions for rectangular cw pulses, frequency- and phase-modulated cw pulses (linear chirp, with Gaussian envelopes, etc.), and perhaps of chief interest, signals in the form of noise pulses, for which are calculated the means and variance of the associated ambiguity functions. 
Chapter V: "Echo Signals": The "echo signals" here are the desired signals reflected from the object of detection (or "research," cf. Chapter I) and received by the omnidirectional receiving array. The emphasis is on Gaussian signal variations imposed on the original, injected signal, and on the calculation of cross-ambiguity functions between these emitted and received (desired) signals. Because of the Gaussian assumptions, a second-moment analysis, involving the calculation of the mean, variance, and correlation function of the cross-ambiguity function, is developed.

Chapter VI: "Marine Reverberation": It is at this stage that the aforementioned assumptions [(i)-(vi) above] regarding the scattering properties of the medium (and its boundaries) and spatial processing (i.e., beam pattern structure) are invoked. Again, the pertinent second-moment theory is constructed for the set of waveforms emitted from the scatterers and picked up by the receiver. This includes an evaluation of the frequency-time cross correlation between the complex envelope of the original signal and the reverberation. For the most part, narrow-band signals are again assumed, although results for a special class of wideband signals are developed. Various envelope statistics are also determined, with correction terms, from which conditions on the limiting Gaussian (or Rayleigh) statistics can be constructed (cf. Contents).

Chapter VII: "Problems of Detecting Echo Signals in the Presence of Interference": This chapter is largely devoted to the calculation of error probabilities of detection (and probabilities of correct detection decisions) for both coherent (i.e., matched-filter, or correlation detection) and incoherent (or energy detection) reception. It is here, of course, that the various first- and second-moment calculations of Chapters $V$ and VI are used as appropriate parameters in the familiar Gauss, Rayleigh, and Rice distributions, which represent the underlying interference models for the different specific conditions of observation here. The chapter concludes with a short extension of these results to multichannel systems.

Chapter VIII: "Elements of the Theory of Statistical Measurement": The subject of this chapter is the development of statistical estimators of (any of) the random 
quantities of interest in the sonar model. Specifically, only quadratic estimators are considered (i.e., those involving a mean-square error calculation, or quadratic costfunction, in the language of statistical decision theory [12]). The mean and variance of this class of estimator are examined and optimum forms are determined, based on smoothing time and a "resolution" or sampling interval for the random magnitude under study. An adaptive optimization procedure applied to various parameters of the measuring system is also discussed here.

Chapter IX: "Inverse Probability Problems in Sonar": This chapter is devoted to various "inverse" problems in sonar: given the injected and received signals, geometries, etc., what can be deduced about the detailed effects of the inhomogeneous medium? To pursue this it is necessary to determine whether such questions represent "well-posed" or "ill-posed" problems, by establishing whether the "correctness conditions" (existence, uniqueness, and stability of solutions) are obeyed. This the author does by applying these concepts to a series of specific "inverse" problems, where it is desired to determine:

(i) the pdf of scatterer Dopplers

(ii) the spectrum of the Doppler fluctuations (which involves an interesting use of Mellin transforms)

(iii) the (relative) level of the coherent component

(iv) the average number of scatterers

(v) the frequency behavior of the scatterers

(vi) the spatial density of the scatterers

[The key conditions underlying one's ability to obtain the specific results here are: (i), the omnidirectionality (and hence frequency insensitivity) of the acoustic arrays coupling to the medium, and (ii), the short duration of the injected signals, to insure ignorable variations with time (i.e., range) over a typical signal duration in the medium; cf. comments below.] 
The book concludes with a rather extensive list of unsolved problems (most of which still remain candidates for further investigation at this date) and a variety of recommended steps toward developing a program for the systems engineering of experimental research in the (active) sonar area, where systems of greater complexity can be studied. A selected bibliography and set of references (selectively expanded by us for this edition) completes the book.

The statistical model of reverberation used in this work is that developed earlier in the so-called FOM (Faure [33], 01'shevskii [14], Middleton [35, 39]) theory, and is thus subject to the limitations of that theory. These are principally: (1), the assumption of independent scattering, with a consequent neglect of multiple scatter effects; and [also stemming from (1)], (2), omission of spatially distributed statistical effects, particularly at boundaries, such as the directional, as well as temporal, correlations of the ocean wave surface. The latter are the main components of the classical, or continuum, "wave models" (for example, $[31,42-44,64])$, which, however, do not yield the independent specular-point or Poissonian component ("speckle" in optical scatter pheonomena) which is the basic scattering mechanism of the FOM models employed here.

Thus, although FOM models appear satisfactory for (weak) volume inhomogeneities, such models are incomplete when interface (wave surface and/or bottom) scattering is involved, since they neglect the random scattering component associated with the continuous portion of the random interfaces, which even at high frequencies can make a noticeable contribution to the total reverberation. On the other hand, the classical continuum and FOM theories can each account (albeit differently in detail) for possible specular (i.e., coherent) components reflected from the interfaces. Moreover, both the FOM and classical "wave models" can be shown to be coexisting subsets of a more general and profound scattering theory, which includes multiple-scattering, intermodal interactions (e.g., surface $\$$ volume scatter), and the global spatio-temporal history of the pertinent scattering operators in the associated Langevin equations of the propagating fields, all from a viewpoint which combines the appropriate physical structure (wave and boundary equations, and initial conditions, geom- 
etry, etc.) with communication-theoretic concepts and statistical methods; cf. Middleton [63].

Other acknowledged limitations on the scope of the treatment here are the assumption of Gaussian statistics (as the limit of a large number of independent scattering events perceived at the receiver) and the use of omnidirectional acoustic arrays, which are also required to be frequency insensitive. The former is reasonable as long as the number of effective scatterers affecting the receiver is large, and no few, particular scattering elements respond more strongly than the rest to the incident field. When this is not the case, the applicability of a secondmoment theory, characteristic of Gaussian processes, breaks down, and (as the author also notes) higher moments of even very high order are required to help specify receiver performance. The latter assumption (of omnidirectional arrays) makes the explicit treatment of inverse problems (given in Chapter IX) analytically practicable.

The usual apertures employed by most sonar systems, however, are directional (and frequency sensitive [69]), and thus, with them, the extraction of scatterer structure and properties involves a spatial deconvolution of the scattered field and these coupling apertures, an analytically nontrivial task. Similar considerations affect the analytic forms of the various injected and received signals $[13,35,39]$ generally.

Another limitation is the assumption of zero-gradient media $(\nabla c=0)$, which, while pedagogically no impediment to the basic approach illustrated here, does confine the applicability of the specific results to a rather restricted class of physical situations: high frequency and short ranges, or special ocean situations where $\nabla c=0$ for whatever frequencies and ranges may be selected [42].

Finally, other important associated topics, such as array processing $[13,40,41,48,60,69]$, the specific role of the ocean surface (and bottom) in reverberation $[41,43,44,63,65]$, and the effects of ambient noise fields, are not considered, in keeping, however, with the author's specified intent to illustrate the use of statistical methods in active sonar in a work of convenient and moderate size. 
Apart from the clear exposition and organization, and in addition to the specific results of Chapters VI and VII (particularly those involving "noise" signals), there are a number of highlights which should be of particular interest to the reader. These appear for the most part as the topics of Chapters VIII and IX. Also, the concluding section offers an interesting (and still largely unachieved) program of pertinent future studies. As a useful exposition of the statistical approach in this field, the book fulfills its main purpose, along with a variety of specific results not readily available in book form elsewhere.

The editor (D.M.) has modified the translation with only minor changes, principally to adjust terminology here and there to Western usage (e.g., "ambiguity functions" for "indeterminacy functions," "quantity" or "estimate" for "valuation," etc.); he has corrected a number of obvious misprints, and has incorporated the author's recent (1976) corrections to the original Russian edition. Besides this Foreword, and a number of explanatory footnotes throughout the text, the editor has added a selected set of pertinent references, which were not available to the author at the time, and which it is hoped will further enhance the book's interest and utility.

David Middleton

New York, October 1977 


\section{PREFACE TO THE AMERICAN EDITION}

This book was conceived as an illustration of the use of statistical methods in active sonar, which is one of the effective ways of exploring the ocean using statistical methods. In keeping with the restricted scope of the book, only isolated questions in the statistical theory of sonar were considered. Undoubtedly the body of problems which might be successfully resolved by means of statistical methods is significantly broader, and a great deal remains to be done.

The author is indebted to Plenum Publishing Corporation for the interest shown in this book, the more so because it is the second book by the author to be issued in the USA by this publisher (the monograph "Characteristics of Sea Reverberation" was published in 1967).

I consider it a pleasant duty to express my gratitude to the initiator of the present publication -- David Middleton. Personal contact with Professor Middleton has not only been useful in a scientific sense, but also has given me great pleasure.

V. V. 01'shevskii

Moscow, March 1976 


\section{PREFACE TO THE RUSSIAN EDITION}

Sonar methods are presently enjoying increasingly greater application. For example, active sonar systems are being used to study various physical characteristics of the oceans, seas, and river basins, to search for minerals located under water, to search for fish and other marine animals, and so on. Such diverse, effective uses of sonar systems demand considerable development of the theory and the engineering behind these systems, chiefly the development of probability and statistical methods in sonar.

This book is devoted to a presentation of selected problems in the use of probability and statistical methods in sonar. These methods play a decisive role in the formulation and conduct of quantitative experimental investigations for the purpose of comparing statistical measurements with probability models of the objects being studied.

The contents of the book is presented in the following order.

The introductory chapter, Chapter I, discusses the sonar model and its principal statistical aspects.

Chapter II is devoted to the development of quantitative methods for assessing the quality of experimental sonar research. Attention is turned to the need for deve1oping probability models of the subjects of research and for making statistical measurements in experiments.

Various methods for constructing probability models of sonar signals, by which the subjects of research can be described, are examined in Chapter III. Most of the attention is devoted to the development of phenomenological probability models -- canonical, parametric, and constructive. 
Chapter IV contains a classification and review of most of the emitted signals encountered in practice.

The characteristics of echo signals satisfying various probability models of distortion stemming both from reflection of sound waves by objects being detected and from the effects of the conditions imposed by underwater observation are examined in Chapter $V$. The characteristics of echo signals are compared through spectrum correlation.

Chapter VI presents a probability analysis of reverberatory signals for the case in which acoustic waves are scattered by discrete irregularities in the water medium.

Chapter VII is devoted to a study of the detection of echo signals in the presence of interference. Information processing methods typical of sonar are analyzed.

Chapter VIII presents some concepts of modern statistical measurement theory as a means for obtaining quantitative data on the probability characteristics of random processes. Methods for optimizing statistical measurements are also discussed here.

Various inverse probability problems encountered in sonar, which involve determining the properties of scatterers on the basis of the structure of received signals, are examined in Chapter IX.

The author confesses that the book offered to the reader does not include some interesting, pressing problems of statistical theory and sonar. Its chief purpose is to illustrate the effectiveness of a statistical approach for solving some important problems of both theoretical and practical importance.

The author is grateful to the reviewers of the book, V. I. Klyachkin and A. K. Novikov, for their useful remarks, and to T. P. Zlobina and V. A. Pulle for their assistance in preparing the manuscript. 


\section{CONTENTS}

Chapter I. Introductory Remarks . . . . . . . . . 1

$\S 1$. Some Definitions . . . . . . . . . . . . . . 1

§2. The Sonar Model . . . . . . . . . . . . . . 3

§3. Sonar and Radar . . . . . . . . . . . . . 8

§4. Statistical Problems of Sonar . . . . . . . . 11

Chapter II. Procedural Fundamentals of Experimental

Research in Sonar... . . . . . 17

§5. Principal Tasks of Experimental Research . . . 17

§6. The Structural Diagram of Quantitative

Experimental Research . . . . . . . . . 19

§7. Discrimination Functional of the Probability

Characteristic and Its Statistical Estimate . 23

§8. The Base Model . . . . . . . . . . . . . 26

§9. Interpretation of Experimental Results . . . . 27

§10. Objects of Experimental Research in Sonar . . . 30

Chapter III. Probability Models of Hydroacoustic

Signals .......... 33

§11. Varieties of Mode1s . . . . . . . . . . . . 33

§12. A Priori Information and Probability

Models . . . . . . . . . . . . . 37

§13. Methods for Describing Hydroacoustic Signals

in Terms of Probability . . . . . . . . . 38

§14. Classification of Hydroacoustic Signals . . . . 41

§15. Canonical Models ... . . . . . . . . . . 44

§16. Parametrical Models .. . . . . . . . . . . 47

§17. Additive-Multiplicative Constructive Mode1s . . 47

$\S 18$. Models in the Form of Oscillations with

Random Amplitude and Phase . . . . . . . . 48

§19. Complex Representation of Signals . . . . . . . 48 
Chapter IV. The Emitted Signals . . . . . . . . 51

§20. Signal Classification . . . . . . . . . . . 51

§21. Complex Representation of Emitted Signals . . . 53

§22. Ambiguity Function and Diagrams . . . . . . . . 55

§23. Signals with Sinusoidal Occupation . . . . . 59

§24. Frequency-Modulating Signals . . . . . . . . . 62

§25. Noiselike Signals . . . . . . . . . . . 65

Chapter V. Echo Signals . . . . . . . . . . . 71

§26. Classification of Objects of Research and the Conditions under Which They Are Observed . . 71

§27. Representation of Echo Signals and Their Probability Characteristics . . . . . . . 74

§28. The Mode1 of an Echo Signal of Known Form with Constant Lag and Change in Time Scale . . . 77

§29. Echo Signal with Fluctuating Amplitude . . . . 81

§30. Echo Signal Consisting of an Additive Mixture of a Signal of Known Form and a Random Component .. . . . . . . . . . . 83

§31. Echo Signal with Random Amplitude Modulation . 88

§32. Echo Signal with Random Phase Modulation . . . 92

§33. Echo Signal with Random Frequency Modulation . 95

§34. Model of an Echo Signal in the Form of a Sum of Elementary Signals . . . . . . . . . . 101

Chapter VI. Marine Reverberation . . . . . . . . 107

§35. Classification of Reverberatory Signals . . . . 107

§36. Discrete Canonical Model of Reverberation . . . 108

§37. Complex Representations . . . . . . . . . . . . 110

§38. Characteristics of the Random Parameters of Elementary Scattered Signals . . . . . 112

§39. Average Value of Reverberation . . . . . . . . 112

\$40. Autocorrelation Function for the Complex Envelope of Reverberation . . . . . . . . 115

§41. Energy Spectrum of the Complex Envelope of Reverberation . . . . . . . . . . 117

§42. Evaluation of the Frequency-Time CrossCorrelation Function for the Complex Envelopes of the Emitted Signal and Reverberation . . . 119 
§43. Generalization for the Case of Elementary

Scattered Signals of Different Forms

§44. Space-Time Correlation of Reverberation when Wideband Signals Are Emitted . . . . 123

§45. One-Dimensional Semi-invariants . . . . . . . 130

§46. Probability Distributions for Instantaneous Values of Reverberation and Its Envelope . . 133

Chapter VII. Problems of Detecting Echo Signals in the Presence of Interference . . .

§47. Specific Features of Detecting Echo Signals in Sonar . . . . . . . . . . . . . . 147

§48. Probability Characteristics of Detection . . . 149

\$49. Typical Probability Distributions of the Output Effect ......... . . . . 151

$\S 50$. Incoherent Detection of Simple Echo Signals with a Linear Detector . . . . . . . . . 155

§51. Incoherent Detection of a Noiselike Echo Signal with Quadratic Detection and Smoothing ("Energy Detection") . . . . . .

§52. Coherent Detection of a Noiselike Echo Signal Using Cross-Correlation Processing . . . . .

§53. The Effect of Reverberation Interference on Echo Signal Detection . . . . . . . . 168

§54. Detection with Multichanne1 Systems, and Discussion of Results . . . . . . . . . .

Chapter VIII. Elements of the Theory of Statistical Measurement . . . . . . . .

§55. Basic Stages of Statistical Measurements . . 179

§56. Statistical Measurement Errors . . . . . . . . 182

§57. Formation of a Statistical Estimate . . . . . 184

§58. Statistical Measurement Errors of the

Characteristics of Nonstationary

Homogeneous Random Processes . . . . . . 185

\$59. Optimization of the Parameters of the

Statistical Measuring System . . . . . 193

§60. Adaptive Optimization . . . . . . . . . . . 195 
Chapter IX. Inverse Probability Problems in Sonar. . 199

§61. Direct and Inverse Problems . . . . . . . . 199

§62. Correctness Conditions . . . . . . . . . . . 202

§63. Determination of the Probability Density for 206

§64. Determination of the Spectrum of the

Fluctuations in the Rate of Movement of

Scatterers . . . . . . . . . . . . 209

\$65. Determination of the Relative Leve1 of

Coherent Scattering . . . . . . . . . 219

§66. Determination of the Average Number of

Scatterers . . . . . . . . . . . 222

§67. Determination of the Frequency Properties

of Scatterers . . . . . . . . . . . . 224

§68. Determination of the Spatial Distribution

of Scatterers . . . . . . . . . . 225

Conclusion . . . . . . . . . . . . . . . 229

Bibliography . . . . . . . . . . . . . 235 